\title{
Equivalence between Bell inequalities and quantum Minority game
}

\author{
Adrian P. Flitney ${ }^{\mathrm{a}, *}$ Maximilian Schlosshauer ${ }^{\mathrm{a}}$ \\ Christian Schmid ${ }^{\mathrm{b}}$ Wieslaw Laskowski ${ }^{\mathrm{c}}$ \\ Lloyd C. L. Hollenberg a,d \\ a School of Physics, University of Melbourne, VIC 3010, Australia \\ ${ }^{\mathrm{b}}$ Max-Planck-Institute for Quantum Optics, D-85748 Garching, Germany \\ ${ }^{\mathrm{c}}$ Institute of Theoretical Physics and Astrophysics, University of Gdańsk, \\ PL-80-952 Gdańsk, Poland \\ ${ }^{\mathrm{d}}$ Centre of Excellence for Quantum Computer Technology, University of \\ Melbourne, Australia
}

\begin{abstract}
We show that, for a continuous set of entangled four-partite states, the task of maximizing the payoff in the symmetric-strategy four-player quantum Minority game is equivalent to maximizing the violation of a four-particle Bell inequality with each observer choosing the same set of two dichotomic observables. We conclude the existence of direct correspondences between (i) the payoff rule and Bell inequalities, and (ii) the strategy and the choice of measured observables in evaluating these Bell inequalities. We also show that such a correspondence between Bell polynomials (in a single plane) and four-player, symmetric, binary-choice quantum games is unique to the four-player quantum Minority game and its "anti-Minority" version. This indicates that the four-player Minority game not only plays a special role among quantum games but also in studies of Bell-type quantum nonlocality.
\end{abstract}

Key words: Quantum game theory; Bell inequalities; Minority game PACS: 03.67.-a, 02.50.Le

\footnotetext{
* Corresponding author.

Email address: aflitney@unimelb.edu.au (Adrian P. Flitney).
} 


\section{Introduction}

The experimentally observed violations of Bell inequalities reflect deep aspects of realism and locality in nature [12] supporting the quantum-mechanical description of correlations between spatially separated systems. Quantum game theory, on the other hand, is an active branch of quantum information theory yet seems far removed from such physical truths, recent implementations notwithstanding [3,4]. In this work we show for the first time a remarkable equivalence between aspects of these seemingly disparate corners of quantum theory. While one may expect a correspondence at some level between quantum game theory and non-classical correlations through Bell inequalities [5], the exact equivalence we have uncovered is rather surprising.

Using an entangled state resource that utilizes a superposition of the GHZ state and products of EPR pairs, we demonstrate equivalence between the optimal symmetric strategy payoffs for a quantum Minority game (QMG) and the violation of the Mermin-Ardehali-Belinskii-Klyshko (MABK) inequality [6,7,8] for the initial state. Although previous publications have drawn a comparison between so-called non-local games and Bell inequalities [9,10, non-local games are cooperative tasks for teams of remote players and not games in the von Neumann sense. Our results are the first direct equivalence between the payoffs in a competitive quantum game and violation of Bell inequalities.

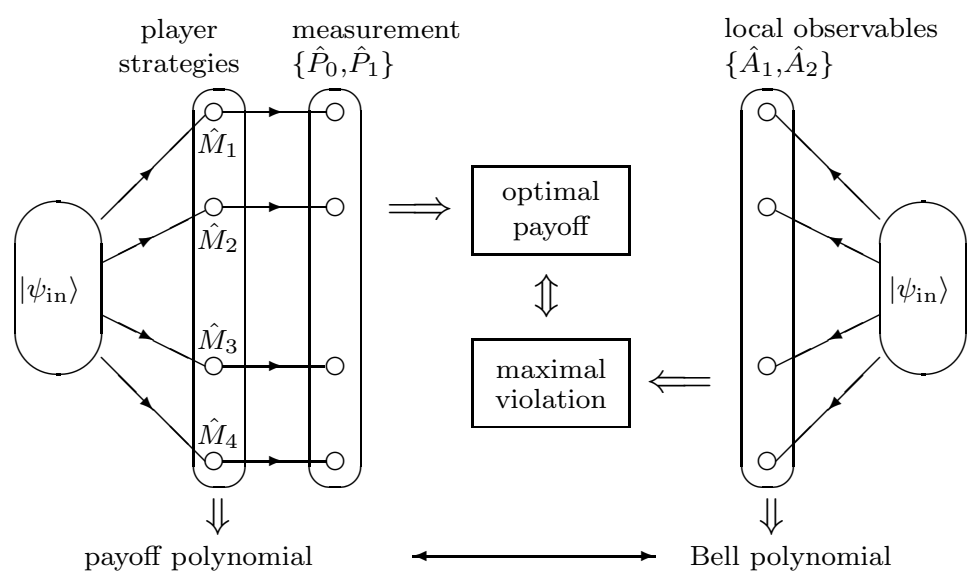

Fig. 1. Schematic showing the equivalences between (left) a four-player quantum Minority game (QMG) and (right) a four-partite Bell inequality. In the QMG, each player acts on one qubit from an entanglement resource $\left|\psi_{\text {in }}\right\rangle$ with a local (unitary) strategy $\hat{M}_{i}$, followed by a projective measurement in the computational basis. The payoff matrix and the chosen strategies determine a payoff polynomial. In the Bell inequality, observers choose local observables $\left\{\hat{A}_{1}, \hat{A}_{2}\right\}$ to make measurements on each qubit of the same entanglement resource. Optimizing the payoff in the game is equivalent to maximizing the violation of the MABK-type Bell inequality. 
The classical Minority game [11] is a simple multi-player game for studying coordination amongst a group of agents in the absence of communication. In each round, the agents must independently select one of two options, labeled ' 0 ' and ' 1 '. Those that select the option chosen by the minority win and are awarded a payoff of one unit, while the others receive zero payoff. In a one-off classical game the players can do no better than using the mixed strategy of selecting each of the two options with equal probability. When there are an even number of players this can result in no minority and hence zero payoff to all players.

While game theory is the mathematical language of competitive (classical) interactions, quantum game theory is the natural language to consider competitive situations in a quantum information setting. One exploits the classical framework as a base for finding new ways of understanding and using entanglement in this context. Although playing a game using entanglement as a resource is not the same as playing the underlying classical game, sharing entanglement is less strong than explicit cooperation. In quantum versions of the Minority game [12,13,14,15], for even numbers of players it has been shown 12]13 that the probability of getting no winners in the final state can be reduced, to the benefit of all players. For small numbers of players the QMG is amenable to experimental implementation using multi-photon entangled states [4].

Quantization of the Minority game proceeds as follows and is shown schematically on the left-hand side of Fig. 1. Each of $N$ players receives one qubit from a known entangled state. The players' strategy is their choice of local unitary operator to apply to their qubit,

$$
\hat{M}\left(\theta, \beta_{1}, \beta_{2}\right)=\left(\begin{array}{cc}
e^{\mathrm{i} \beta_{1}} \cos (\theta / 2) & \mathrm{i} e^{\mathrm{i} \beta_{2}} \sin (\theta / 2) \\
\mathrm{i} e^{-\mathrm{i} \beta_{2}} \sin (\theta / 2) & e^{-\mathrm{i} \beta_{1}} \cos (\theta / 2)
\end{array}\right),
$$

where $\theta \in[0, \pi], \beta_{1}, \beta_{2} \in[-\pi, \pi]$. No communication between the players is permitted and coherence is maintained until after the players' actions. Then the qubits are measured in the computational basis and payoffs are awarded using the usual payoff scheme. If the initial state is a GHZ state, the scheme described is equivalent to the protocol of Eisert et al. [16], since the unentangling gate in Eisert's protocol has no effect on the payoffs for the Minority game [12. The scheme is also consistent with the generalized quantum game formalisms of Lee and Johnson [17] and Gutoski and Watrous [18]. 


\section{Pareto optimal strategies and Bell inequalities}

In the current context we will be concerned with the Pareto-optimal (PO) strategy profile, one from which no player can improve their result without another being worse off. We only consider the situation where all players use the same strategy, since asymmetric strategy profiles are problematic to achieve in the absence of communication. The symmetric PO result gives the maximal payoff that is fair to all players.

Existing works have concentrated on using a GHZ state as the entanglement resource for the game, however, we shall consider the more general initial state

$$
\left|\psi_{\mathrm{in}}\right\rangle=\alpha|\mathrm{GHZ}\rangle+\sqrt{1-\alpha^{2}}|\mathrm{EPR}\rangle_{\mathrm{AB}} \otimes|\mathrm{EPR}\rangle_{\mathrm{CD}}
$$

where $|\mathrm{GHZ}\rangle \equiv(|0000\rangle+|1111\rangle) / \sqrt{2},|\mathrm{EPR}\rangle \equiv(|01\rangle+|10\rangle) / \sqrt{2}$, and $\alpha \in$ $[0,1]$. This state can be created experimentally for arbitrary $\alpha$ using photons produced from down conversion [4, 19].

Below we show that the payoff observable for the Minority game, when transformed by the PO strategy profile, gives rise to a Bell-like polynomial that, when evaluated for the initial state Eq. (2), is identical for all $\alpha$ (up to an arbitrary scaling factor) to the maximal value of the MABK Bell polynomial.

In the game, the state prior to measurement is $\left|\psi_{\text {final }}\right\rangle=\hat{M}_{1} \otimes \hat{M}_{2} \otimes \hat{M}_{3} \otimes$ $\hat{M}_{4}\left|\psi_{\text {in }}\right\rangle$, where the $\hat{M}_{i}$ are the strategies chosen by the players. For symmetric strategy profiles only the difference between the phases $\beta_{1}, \beta_{2}$ of Eq. (1) is relevant. We shall therefore, without loss of generality, set $\beta \equiv \beta_{1}=-\beta_{2}$. A necessary and sufficient condition for $\hat{M}\left(\theta^{*}, \beta^{*},-\beta^{*}\right)$ to be a symmetric PO strategy is $\left\langle \$\left(\hat{M}\left(\theta^{*}, \beta^{*},-\beta^{*}\right)^{\otimes 4}\right)\right\rangle \geq\left\langle \$\left(\hat{M}(\theta, \beta,-\beta)^{\otimes 4}\right)\right\rangle \forall \theta, \beta$, where $\$$ represents the payoff to any one of the four players for the indicated strategy profile. When all players select the strategy $\hat{M}(\theta, \beta,-\beta)$ for some $\theta, \beta$ to be determined, the average payoff to each player is $\langle \$\rangle=\frac{\sin ^{2} \theta}{32}\left[8-2 \alpha^{2}+\right.$ $8 \alpha \sqrt{2-2 \alpha^{2}} \cos 4 \beta-2 \alpha^{2} \cos 8 \beta+2\left(4-3 \alpha^{2}\right) \cos 2 \theta+8 \alpha \sqrt{2-2 \alpha^{2}} \cos 4 \beta \cos 2 \theta+$ $2 \alpha^{2} \cos 8 \beta \cos 2 \theta$ ]. A local maximum or minimum in the value of the payoff will have $d\langle \$\rangle / d \theta=d\langle \$\rangle / d \beta=0$. From these conditions we find the strategy that maximizes the payoff to be

$$
\begin{array}{ll}
\hat{M}_{>}=\frac{1}{\sqrt{2}}\left(\begin{array}{cc}
\mathrm{e}^{\mathrm{i} \pi / 8} & \mathrm{ie}^{-\mathrm{i} \pi / 8} \\
\mathrm{ie}^{\mathrm{i} \pi / 8} & \mathrm{e}^{-\mathrm{i} \pi / 8}
\end{array}\right) & \text { for } \alpha \geq \sqrt{\frac{2}{3}}, \\
\hat{M}_{<}=\left(\begin{array}{cc}
\cos (\pi / 8) & \mathrm{i} \sin (\pi / 8) \\
\mathrm{i} \sin (\pi / 8) & \cos (\pi / 8)
\end{array}\right) & \text { for } \alpha \leq \sqrt{\frac{2}{3}},
\end{array}
$$




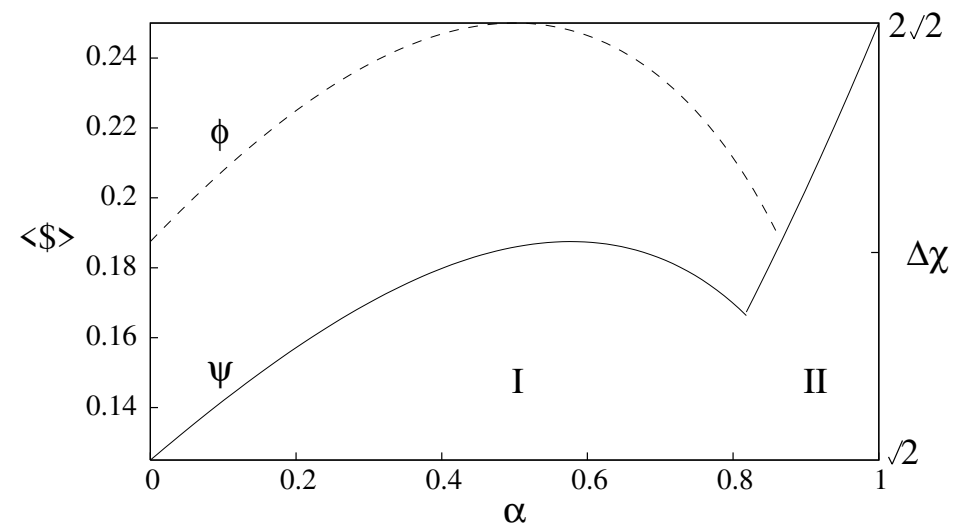

Fig. 2. Left scale: Pareto optimal payoffs $\left\langle \$_{<}\right\rangle$(region I) and $\left\langle \$_{>}\right\rangle$(region II) in a four-player quantum Minority game with the initial states $\left|\psi_{\text {in }}\right\rangle$ [Eq. (2)] and $\left|\phi_{\text {in }}\right\rangle$ [Eq. (15)]. Right scale: Maximal violation of the MABK Bell equality Eq. (10) for the same states. For measurement schemes, see text. The quantum fulcrum between regions I and II occurs at $\alpha=\sqrt{\frac{2}{3}}$ for $\left|\psi_{\text {in }}\right\rangle$ and $\alpha=\sqrt{\frac{3}{4}}$ for $\left|\phi_{\text {in }}\right\rangle$.

with expected payoffs $\left\langle \$_{>}\right\rangle=\frac{1}{4} \alpha^{2}$ and

$$
\left\langle \$_{<}\right\rangle=\underbrace{\frac{1}{16}}_{\hat{I} \hat{I} \hat{I} \hat{I}, \ldots}+\frac{1}{16}(\underbrace{1-\alpha^{2}}_{-\hat{Y} \hat{Y} \hat{Z} \hat{Z}, \ldots}+2 \underbrace{\sqrt{2} \alpha \sqrt{1-\alpha^{2}}}_{-\hat{Y} \hat{Z} \hat{Y} \hat{Z}, \ldots}),
$$

where the contributions from the corresponding measurement correlations $\left\langle\psi_{\text {in }}|\cdots| \psi_{\text {in }}\right\rangle$ are explicitly given under the braces [see Eq. (7)]. The payoffs as a function of $\alpha$ are shown in Fig. 2. At $\alpha=\sqrt{\frac{2}{3}}$, we find that, disregarding phases, the states $\hat{M}_{>}^{\otimes 4}\left|\psi_{\text {in }}\right\rangle$ and $\hat{M}_{<}^{\otimes 4}\left|\psi_{\text {in }}\right\rangle$ contain exactly the same terms, all with identical prefactors. Hence $\alpha=\sqrt{\frac{2}{3}}$ is the boundary between the EPRand the GHZ-dominated regions. This point is a fulcrum in the quantum state where there is a switch in optimal strategy from $\hat{M}_{<}$to $\hat{M}_{>}$.

Let us write down a payoff observable $\hat{\$}$ as a sum of projection operators on the winning states,

$$
\begin{aligned}
\hat{\$}= & \frac{1}{4}\left(\hat{P}_{0}^{(1)} \otimes \hat{P}_{1}^{(2)} \otimes \hat{P}_{1}^{(3)} \otimes \hat{P}_{1}^{(4)}+\right.\text { permutations } \\
& \left.+\hat{P}_{0}^{(1)} \otimes \hat{P}_{0}^{(2)} \otimes \hat{P}_{0}^{(3)} \otimes \hat{P}_{1}^{(4)}+\text { permutations }\right),
\end{aligned}
$$

where $\hat{P}_{j}^{(k)}$ is the projector $|j\rangle\langle j|, j=0,1$, for the $k$ th qubit. The average payoff is then given by

$$
\langle \$\rangle=\left\langle\psi_{\text {in }}\left|\hat{M}^{\dagger \otimes 4} \hat{\$} \hat{M}^{\otimes 4}\right| \psi_{\text {in }}\right\rangle .
$$

Using $\hat{M}_{<}^{\dagger} \hat{P}_{0 / 1} \hat{M}_{<}=\frac{1}{2}\left(\hat{I} \pm \hat{B}_{z y}\right)$ with $\hat{B}_{z y} \equiv \frac{1}{\sqrt{2}}(\hat{Z}-\hat{Y})$, the payoff observable 
Eq. (5) transformed by the optimal strategy for $\alpha<\sqrt{\frac{2}{3}}$ is

$$
\begin{aligned}
& \hat{\$}_{<} \equiv \hat{M}_{<}^{\dagger \otimes 4} \hat{\$} \hat{M}_{<}^{\otimes 4} \\
&=\frac{1}{32}(-\hat{Z} \hat{Z} \hat{Z} \hat{Z}+\hat{Z} \hat{Z} \hat{Z} \hat{Y}+\hat{Z} \hat{Z} \hat{Y} \hat{Z}-\hat{Z} \hat{Z} \hat{Y} \hat{Y} \\
& \quad+\hat{Z} \hat{Y} \hat{Z} \hat{Z}-\hat{Z} \hat{Y} \hat{Z} \hat{Y}-\hat{Z} \hat{Y} \hat{Y} \hat{Z}+\hat{Z} \hat{Y} \hat{Y} \hat{Y} \\
& \quad+\hat{Y} \hat{Z} \hat{Z} \hat{Z}-\hat{Y} \hat{Z} \hat{Z} \hat{Y}-\hat{Y} \hat{Z} \hat{Y} \hat{Z}+\hat{Y} \hat{Z} \hat{Y} \hat{Y} \\
&\quad-\hat{Y} \hat{Y} \hat{Z} \hat{Z}+\hat{Y} \hat{Y} \hat{Z} \hat{Y}+\hat{Y} \hat{Y} \hat{Y} \hat{Z}-\hat{Y} \hat{Y} \hat{Y} \hat{Y}) \\
& \quad+\frac{1}{8} \hat{I} \hat{I} \hat{I} \\
&=-\frac{1}{8}\left(\hat{B}_{z y}\right)^{\otimes 4}+\frac{1}{8} \hat{I}^{\otimes 4} .
\end{aligned}
$$

The first term can be considered to arise as a result of quantum effects while the identity term is the classical part, since $\frac{1}{8}$ is just the average payoff in a classical (unentangled) Minority game when the players select the optimal strategy. Similarly, $\hat{M}_{>}^{\dagger} \hat{P}_{0 / 1} \hat{M}_{>}=\frac{\hat{I} \pm \hat{B}_{x y}}{2}$, with $\hat{B}_{x y}=\frac{1}{\sqrt{2}}(\hat{X}-\hat{Y})$. This expression coincides with that for the strategy $\hat{M}_{<}$with the simple substitution $\hat{Z} \leftrightarrow \hat{X}$. Thus for $\hat{\$}_{>}$we get expression Eq. (7) with $\hat{Z}$ replaced by $\hat{X}$.

Because of the similarity between Eq. (7) and a four-particle Bell polynomial we write Eq. (6) as a payoff polynomial

$$
\begin{aligned}
\chi_{\text {payoff }}= & 4-E(1111)+E(1112)+E(1121)-E(1122) \\
& +E(1211)-E(1212)-E(1221)+E(1222) \\
& +E(2111)-E(2112)-E(2121)+E(2122) \\
& -E(2211)+E(2212)+E(2221)-E(2222),
\end{aligned}
$$

where we have scaled Eq. (6) by a factor of 32 for simplicity. Here $E\left(k_{1}, k_{2}, k_{3}, k_{4}\right)=$ $\left\langle\psi_{\text {in }}\left|\hat{A}_{k_{1}} \otimes \ldots \otimes \hat{A}_{k_{4}}\right| \psi_{\text {in }}\right\rangle$ with $k_{i} \in\{0,1\}$. In the case of the QMG, the observables $\left\{\hat{A}_{1}, \hat{A}_{2}\right\} \equiv\{\hat{X}, \hat{Y}\}$ for $\alpha>\sqrt{\frac{2}{3}}$ and $\{\hat{Z}, \hat{Y}\}$ for $\alpha<\sqrt{\frac{2}{3}}$, give $\chi_{\text {payoff }}=8 \alpha^{2}$ and $\chi_{\text {payoff }}=4-2 \alpha^{2}+4 \alpha \sqrt{2-2 \alpha^{2}}$, respectively, i.e., the payoffs scaled by the factor of 32 , as expected.

Now consider a four-particle MABK Bell polynomial, which can be written as 6, 7,8

$$
\begin{aligned}
\chi_{\mathrm{MABK}}= & -E(1111)-E(1112)-E(1121)+E(1122) \\
& -E(1211)+E(1212)+E(1221)+E(1222) \\
& -E(2111)+E(2112)+E(2121)+E(2122) \\
& +E(2211)+E(2212)+E(2221)-E(2222) .
\end{aligned}
$$


A local realistic theory must satisfy the Bell inequality

$$
\left|\chi_{\mathrm{MABK}}\right| \leq 4
$$

This inequality is maximally violated (by a factor of $\Delta \chi=2 \sqrt{2}$ ) by the GHZ state if the first three observers measure in the $\{\hat{X}, \hat{Y}\}$ basis and the fourth observer in the $\left\{\frac{1}{\sqrt{2}}(\hat{X}-\hat{Y}), \frac{1}{\sqrt{2}}(\hat{X}+\hat{Y})\right\}$ basis. Alternately, following [20], maximal violation is obtained if all observers measure in the ba$\operatorname{sis}\{\cos (-\pi / 16) \hat{X}+\sin (-\pi / 16) \hat{Y}, \cos (7 \pi / 16) \hat{X}+\sin (7 \pi / 16) \hat{Y}\}$. These measurement schemes give maximal violation for the state in Eq. (21) provided $\alpha \geq \sqrt{\frac{2}{3}}$. For $\alpha \leq \sqrt{\frac{2}{3}}$, maximal violation is obtained with the same measurement scheme except with $\hat{X}$ replaced with $\hat{Z}$. The violation $\Delta \chi$ of the MABK inequality Eq. (10) versus $\alpha$ is given in Fig. 2.

A comparison of Eq. (8) and Eq. (9) shows a striking similarity between the two expressions. They only differ in the distribution of relative signs $( \pm)$ associated with each term $E(\ldots)$. Remarkably, in the context of the rules and optimal strategies of the QMG, the payoff polynomial Eq. (8) evaluated with the optimal-strategy measurement schemes $\{\hat{X}, \hat{Y}\}^{\otimes 4}$ (for $\alpha \geq \sqrt{\frac{2}{3}}$ ) and $\{\hat{Z}, \hat{Y}\}^{\otimes 4}$ (for $\alpha \leq \sqrt{\frac{2}{3}}$ ) for the initial state Eq. (2) has the same value as the MABK polynomial Eq. (9) evaluated with these schemes and this initial state. In a local realistic theory, the absolute value of both polynomials is always $\leq 4$. Since we have already shown that the strategies Eq. (3) are optimal, we can infer that, for a symmetric strategy profile, the dichotomic observables $\{\hat{X}, \hat{Y}\}^{\otimes 4}$ (if $\alpha \geq \sqrt{\frac{2}{3}}$ ) and $\{\hat{Z}, \hat{Y}\}^{\otimes 4}$ (if $\alpha \leq \sqrt{\frac{2}{3}}$ ) give the maximum violation of the Bell inequality defined by Eq. (8), i.e., $\left|\chi_{\text {payoff }}\right| \leq 4$. Given the continuous spectrum of initial states Eq. (2), optimizing the payoff in the QMG thus corresponds to maximizing the violation of the Bell inequality defined by Eq. (8). This observation directly relates the four-player QMG discussed in this paper to Bell inequalities.

We now show that the Minority game is effectively unique amongst fourplayer, symmetric, binary-choice games in its connection to Bell inequalities. We restrict ourselves to considering Bell polynomials with measurement in a single plane (i.e., $\hat{X} \hat{Y}, \hat{X} \hat{Z}, \hat{Y} \hat{Z}$ ) though there are Bell inequalities defined on two planes that may also give rise to a connection with quantum games. We assume that all players choose the same strategy and consider a general payoff observable

$$
\hat{\$}=\sum_{j_{1}, j_{2}, j_{3}, j_{4}=0,1} c_{j_{1} j_{2} j_{3} j_{4}} \bigotimes_{k=1}^{4} \hat{P}_{j_{k}}^{(k)} .
$$

Application of a strategy $\hat{M}(\theta, \beta,-\beta)$ to each qubit transforms the projection 
operators $\hat{P}_{j_{k}}^{(k)}$ as

$$
\begin{aligned}
\hat{M}^{\dagger} \hat{P}_{j} \hat{M}= & \frac{1}{2} \hat{I}+\left(\cos ^{2} \frac{\theta}{2}-\frac{1}{2}\right) \hat{Z} \\
& +(-1)^{j} \frac{1}{2} \sin \theta(\sin 2 \beta \hat{X}-\mathrm{i} \cos 2 \beta \hat{Y}) .
\end{aligned}
$$

To correspond to a single-plane Bell polynomial, we eliminate one of the terms $\hat{X}, \hat{Y}, \hat{Z}$ with an appropriate choice of $\theta, \beta$. This can be done by selecting,

$$
\begin{aligned}
& \hat{Z}: \quad \theta=\frac{\pi}{2}, \frac{3 \pi}{2}, \quad \beta \text { arbitrary, } \\
& \hat{X}: \quad \theta \text { arbitrary, } \quad \beta=0, \frac{\pi}{2}, \pi, \frac{3 \pi}{2}, \\
& \hat{Y}: \quad \theta \text { arbitrary, } \quad \beta=0, \frac{\pi}{4}, \frac{3 \pi}{4}, \frac{5 \pi}{4} .
\end{aligned}
$$

Next, terms in the polynomial containing identity operators must mutually cancel, save for an ineliminable but trivial term $\hat{I}^{\otimes 4}$. This gives fourteen constraints on the $c_{j_{1} j_{2} j_{3} j_{4}} \mathrm{~s}$, with the last two conditions in Eq. (13) having in

addition $\sum_{j_{1} j_{2} j_{3} j_{4}}=0$. The constraints can be solved in terms of two parameters $a$ and $b$ :

$$
\begin{aligned}
c_{0001} & =c_{0010}=c_{0100}=c_{1000} \\
& =c_{0111}=c_{1011}=c_{1101}=c_{1110}=a \\
c_{0000} & =c_{0011}=c_{0101}=c_{0110} \\
& =c_{1001}=c_{1010}=c_{1100}=c_{1111}=b
\end{aligned}
$$

With $a>0$ and $b<0$ we have the Minority game, while reversing the signs gives the complement, the anti-Minority game, where each player scores $\frac{1}{n}$ when there is no strict minority, $n$ being the number of players. In the case where we use the first of the conditions in Eq. (13) we can have $a$ and $b$ with the same sign, but these games are the trivial ones where either everyone wins, or everyone looses, depending on the sign of $a$.

\section{Extensions}

It is interesting to consider an initial state that, unlike Eq. (2), is completely symmetric with respect to the interchange of any two qubits,

$$
\begin{aligned}
\left|\phi_{\mathrm{in}}\right\rangle=\alpha|\mathrm{GHZ}\rangle+ & \sqrt{\frac{1-\alpha^{2}}{3}}\left(|\mathrm{EPR}\rangle_{\mathrm{AB}} \otimes|\mathrm{EPR}\rangle_{\mathrm{CD}}\right. \\
& \left.+|\mathrm{EPR}\rangle_{\mathrm{AC}} \otimes|\mathrm{EPR}\rangle_{\mathrm{BD}}+|\mathrm{EPR}\rangle_{\mathrm{AD}} \otimes|\mathrm{EPR}\rangle_{\mathrm{BC}}\right) .
\end{aligned}
$$


The boundary between the EPR- and GHZ-dominated regions now occurs at $\alpha=\sqrt{\frac{3}{4}}$, with expected payoffs $\left\langle \$_{>}\right\rangle=\frac{1}{4} \alpha^{2}$ and

$$
\left\langle \$_{<}\right\rangle=\underbrace{\frac{1}{16}}_{\hat{I} \hat{I} \hat{I} \hat{I}, \ldots}+\frac{3}{16}(\underbrace{\frac{2}{3}\left(\sqrt{3} \alpha \sqrt{1-\alpha^{2}}+1-\alpha^{2}\right)}_{-\hat{Y} \hat{Z} \hat{Y} \hat{Z}, \ldots,-\hat{Y} \hat{Y} \hat{Z} \hat{Z}, \ldots}) .
$$

For this higher-symmetry state, the correspondence between the MABK inequality and the PO payoffs remains (dashed line in Fig. 2).

The nature of the correspondence for a larger case is also of interest. We have considered the particular case of a six-player QMG using a state analogous to Eq. Eq. (15). We find that the switch of optimal strategy occurs at $\alpha=\sqrt{\frac{6}{19}}$, with payoffs

$$
\begin{aligned}
& \left\langle \$_{>}\right\rangle=\frac{2+3 \alpha^{2}}{16} ; \\
& \left\langle \$_{<}\right\rangle=\frac{7\left(2-\alpha^{2}\right)}{64} .
\end{aligned}
$$

Following [20], the symmetric measurement schemes that give rise to maximal violation of the MABK inequality for this six-particle state [6,7,8] are $\{\cos (\pi / 24) \hat{X}+\sin (\pi / 24) \hat{Y}, \cos (13 \pi / 24) \hat{X}+\sin (13 \pi / 24) \hat{Y}\}$ for $\alpha \geq \sqrt{\frac{5}{13}}$, and the same with $\hat{X} \rightarrow \hat{Z}$ for $\alpha \leq \sqrt{\frac{5}{13}}$. Although for this particular case the switch in optimal measurement scheme does not occur at the same value of $\alpha$ as the switch in optimal strategy, the payoff and violation curves are qualitatively similar. It will be interesting to explore the trajectory of initialstate entanglement, strategies, and inequalities which may give rise to direct equivalence for higher- $N$ cases.

\section{Conclusions}

We have demonstrated for the first time a direct equivalence between Bell inequalities and quantum games. The symmetric Pareto optimal payoff in a four-player quantum Minority game is equivalent to the violation of the MABK-type Bell inequality for an important class of four-partite entangled states that involve the GHZ state and products of EPR pairs. The payoff scheme for the quantum game combined with the strategies chosen by the players leads to a payoff polynomial that is analogous to the Bell polynomial. For both the optimal payoff in the quantum game and for the Bell inequality there is a quantum fulcrum where there is a switch in preferred strategy (quantum game) or measurement scheme (Bell inequality) corresponding to a change from the EPR- to GHZ-dominated region. 
The equivalence uncovered here is important from the view of both quantum game theory and Bell inequalities. Our result shows that the four-player quantum (anti-) Minority game assumes a special position, since it uses quantum nonlocality to achieve an advantage over the classical case in precisely the same way nonlocality is evidenced through the violation of the Bell inequalities. This also implies that the lessons learned from interpreting the nature of nonlocality in quantum mechanics through the lens of Bell inequalities can be readily applied to advancing our understanding of the discerning features of quantum game theory over its classical counterpart.

\section{Acknowledgments}

This project was supported by the Australian Research Council (ARC). APF and MS are recipients of ARC Postdoctoral Fellowships (project numbers DP0559273 and DP0773169, respectively), and LCLH is the recipient of an ARC Professorial Fellowship (project number DP0770715).

\section{References}

[1] S. Gröblacher et al., Nature 446 (2007) 871.

[2] A. J. Leggett, Found. Phys. 33 (2003) 1469.

[3] R. Prevedel, A. Stefanov, P. Walther, A. Zeilinger, New J. Phys. 9 (2007) 205.

[4] C. Schmid et al., in preparation.

[5] S. J. van Enk, R. Pike, Phys. Rev. A 66 (2002) 024306.

[6] N. D. Mermin, Phys. Rev. Lett. 65 (1990) 1838.

[7] M. Ardehali, Phys. Rev. A 46 (1992) 5375.

[8] A. V. Belinskii, D. N. Klyshko, Physics-Uspekhi 36 (1993) 653.

[9] J. Silman, S. Machnes, N. Aharon, Phys. Lett. A 372 (2008) 3796.

[10] M. Pawłowski, e-print: quant-ph/0710.3881.

[11] D. Challet, Y. C. Zhang, Physica A 246 (1997) 407.

[12] S. C. Benjamin, P. M. Hayden, Phys. Rev. A 64 (2001) 030301(R).

[13] Q. Chen et al., Phys. Lett. A 327 (2004) 98.

[14] A. P. Flitney, L. C. L. Hollenberg, Quant. Inf. Comput. 7 (2007) 111. 
[15] A. P. Flitney, A. D. Greentree, Phys. Lett. A 362 (2007) 132.

[16] J. Eisert, M. Wilkens, M. Lewenstein, Phys. Rev. Lett. 83 (1999) 3077.

[17] C. F. Lee, N. F. Johnson, Phys. Rev. A 67 (2003) 022311.

[18] G. Gutoski, J. Watrous, Proc. 39th Symp. on Theory of Computing (STOC'07), p. 565, 2007.

[19] H. Weinfurter, M. Żukowski, Phys. Rev. A 64 (2001) 010102(R).

[20] R. F. Werner, M. M. Wolf, Phys. Rev. A 64 (2001) 032112. 